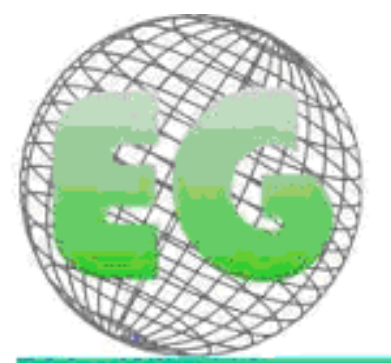

15SN 1695-6) *S:

\title{
EL CUIDADOR Y EL ENFERMO EN EL FINAL DE LA VIDA - FAMILIA Y/O PERSONA SIGNIFICATIVA
}

O CUIDADOR E O DOENTE EM DE FIM DE VIDA - FAMÍLIA E/OU PESSOA SIGNIFICATIVA

\section{${ }^{*}$ Rodrigues Gomes, Ana Margarida}

\author{
*Enfermeira Graduada Servicio de Especialidades | del Hospital Amato Lusitano. Instituto \\ Politécnico de Castelo Branco. Escola Superior de Saúde Dr. Lopes Dias. Portugal. \\ Palabras clave: fin de la vida. Cuidado al final de la vida, cuidador informal.
}

Palavras-chave: fim de vida, cuidado em fim de vida, cuidador informal.Keywords: End of life, Care at the End of Life, Informal Caregiver

\section{RESUMEN}

El presente trabajo pretende abordar el paciente al final de la vida y la familia y/o persona significativa como pilar de su cuidado. Las intervenciones junto a estos cuidadores son esenciales para que el sufrimiento sea minimizado, siendo necesario identificar sus necesidades básicas y la implementación de un plan de intervención ajustado a cada situación en particular.

\section{RESUMO}

O presente trabalho pretende abordar o doente em fim de vida e a família e/ou pessoa significativa como pilar do seu cuidado. As intervenções junto a estes cuidadores são essenciais para que o sofrimento seja minimizado, sendo necessário identificar as suas necessidades básicas e a implementação de um plano de intervenção ajustado a cada situação em particular. 


\begin{abstract}
"Aqueles que tiveram a força e o amor para ficar ao lado de um paciente moribundo com o silêncio que vai além das palavras saberão que tal momento não é assustador nem doloroso, mas um cessar em paz do funcionamento do corpo".
\end{abstract}

\title{
Elisabeth Kübler-Ross ${ }^{1}$, in: Sobre a Morte e o Morrer - apa
}

\section{INTRODUCCIÓN}

El acompañamiento al enfermo en el final de la vida fue tradicionalmente dirigido hacia la muerte, era más importante ocuparse del alma que de los cuidados del cuerpo. Esta concepción de cuidar del "moribundo" fue dando lugar al cuidar "de la persona en el final de la vida", esto es, acompañar en vida, dar importancia a todo lo que altere o perturbe la calidad de vida que le resta. ${ }^{2}$

El cuidado al enfermo en el final de la vida tiene como cuestión primordial determinar cuál es el tratamiento adecuado en función de sus perspectivas biológicas y de sus circunstancias personales y sociales. Los cuidados paliativos son los indicados y tienen como meta proporcionar al enfermo, familia y/o persona significativa condiciones para que alcancen una buena calidad de vida. ${ }^{3,4}$ Además de promover el ajuste del enfermo, familia y/o persona significativa a una nueva realidad, enfrentando de la mejor forma posible la condición de enfermedad terminal, cuyos caminos pasan por el buen control del dolor y de otros síntomas, buena comunicación, apoyo psicosocial y trabajo en equipo.,

La calidad de vida esencialmente existe cuando las aspiraciones de un individuo son alcanzadas y llenadas por su situación actual, siendo necesario disminuir la separación entre las aspiraçiones y aquello que es posible alcanzar. En este contexto los cuidados paliativos se dirigen al enfermo y no a la enfermedad, aceptando la muerte y mejorando la calidad de vida, constituyéndose una alianza entre el enfermo y los prestadores de cuidados, preocupándose más con la "reconciliación" que con la cura. ${ }^{3}$ Compite al que cuida aclarar lo que todavía da sentido en los cuidados y en la relación en el final de la vida, a pesar de las pérdidas, de las alteraciones físicas y psíquicas, de los fracasos terapéuticos y de su impotencia para aliviar completamente el sufrimiento. ${ }^{2,4}$

\section{EL ENFERMO EN EL FINAL DE LA VIDA}

La enfermedad terminal se considera la fase final de numerosas enfermedades crónicas progresivas, cuando han sido agotados todos los tratamientos posibles y se alcanza la irreversibilidad. ${ }^{7}$ En el precurso de la enfermedad el diagnóstico es importante; todavía, se asume como un proceso complejo, que requiere alguna habilidad pues el enfermo en el final de la vida presenta síntomas múltiples y cambiantes. Se revelan como más frecuentes, en esta fase, la dificultad respiratoria (71\%), el dolor moderado a severo (50\%), la incontinencia urinaria y fecal (36\%), la fatiga (18\%) y cerca del $50 \%$ de estos enfermos pasan más tiempo encamados. ${ }^{8}$ Además de la presencia de náuseas y vómitos, estreñimiento, confusión mental, inquietud y anorexia, se evidencia también la pérdida de la fuerza muscular, del apetito y a veces la pérdida de la conciencia, manifestándose también la pérdida o disminución de la capacidad para ingerir líquidos y tomar la medicación por vía oral.

Los últimos días de vida representan la fase de agonía. Se considera, temporalmente, las últimas horas de los últimos 3 ó 5 dias, caso de que sean evidentes las señales o síntomas del proceso de muerte, ${ }^{11}$ destacando como más frecuentes, en las últimas 48 horas de vida, el delirio $(51,7 \%)$, la hemorragia $(20,7 \%)$, la disnea $(17,2 \%)$, el dolor/disnea y la 
agitación/disnea con igual porcentaje de 3,4\%. ${ }^{12} \mathrm{Al}$ mal y al sufrimiento físico sobrevienen el drama psicológico y espiritual, del despojo que significa el morir. ${ }^{7}$

Lo que nos trae algunas cuestiones. ¿Cómo proporcionar los cuidados que merece? ¿Cómo informar acerca de la gravedad de su situación? ¿Qué conductas terapéuticas son más adecuadas? ¿Cuál es el mejor lugar para sus últimos días?

Las respuestas requieren una planificación cuidadosa de los cuidados a prestar a este enfermo, tomando en consideración aspectos como: control del dolor y otros síntomas, delineando y materializando intervenciones terapéuticas (farmacológicas y no farmacológicas) adecuadas a la maximización de los niveles de confort del enfermo, valorizando también la ansiedad y la depresión, el temor y el insomnio. Considerar el apoyo de un equipo multidisciplinar, de modo que fomente y favorezca la comunicación clara en el precurso de la enfermedad, con el enfermo y sus familiares y/o la persona significativa; reconociendo y tratando el estrés del cuidador mitigando las diversas manifestaciones derivadas del proceso de morir. ${ }^{7,9,13}$

\section{EL CUIDADO EN EL FINAL DE LA VIDA}

Cuando se cuida del enfermo en el final de la vida, se debe tener presente, no sólo los princípios éticos y morales que envuelven la relación establecida, sino también considerar que la práctica de la enfermería no se reduce a la mera ejecución de técnicas, pues está implicada una relación interpersonal donde los aspectos emocionales, culturales y espirituales deben ser considerados y valorizados. El enfermo debe ser considerado con todo el respeto que le es debido, como ser humano que es, por lo que personalizar sus necesidades es una prioridad a cimentar por los profesionales de salud, familiares u otros cuidadores, respetándo sus creencias y deseos, dando al proceso de muerte la posibilidad de proseguir su curso, de forma natural, encaminándose para un fin digno y sereno. ${ }^{14,15}$

En esta perspectiva los cuidados paliativos asumen un papel preponderante, evidenciándose en su práctica y aplicación una mayor satisfacción del enfermo (mejor control de síntomas) y familia (control de los niveles de ansiedad). ${ }^{6}$ Pues, su filosofía encuadra directrices respecto a los cuidados a prestar en esta fase, no sólo al enfermo como también en lo que concierne al soporte familiar teniendo en perspectiva un enfermo dependiente de los cuidados de otros y para el que la muerte se sitúa en un horizonte muy próximo. ${ }^{16}$ Lo que constituye un gran desafío, no sólo de los profesionales de salud, sino también de la sociedad. Por eso integrar a la familia, como unidad cuidadora, se vuelve esencial para la planificación y toma de decisiones procurando evitar que las adversidades inherentes a toda la fase de la agonía, no resulten en factores desestabilizantes en el luto sino que, por el contrario, se tornen factores que favorezcan la aceptación de la pérdida. ${ }^{4,10,11,17}$

En la prestación de cuidados debemos seguir algunos principios básicos como: evaluar la situación inicial del enfermo y familia, reconociendo el cuidador principal, el medio ambiente, los medios físicos y personales que puede disponer; determinar las necesidades y problemas generales o específicos, a través de un diagnóstico del enfermo y familia, lo más detallado posible; planificar los cuidados a través de la elaboración de un plano individualizado, en conjunto con el enfermo y familia, compartiendo las decisiones y problemas; ejecutar el plano elaborado, fijando prioridades diarias de cuidados; evaluar los cuidados, tomar en cuenta como estos están siendo realizados, los resultados obtenidos y la percepción de mejoría por parte del enfermo. ${ }^{17,18}$ 
El cuidado al enfermo en el final de la vida debe de ir al encuentro de sus necesidades físicas y psicosociales, adaptándolo a su estado y condición global a cada evaluación.

\section{NECESIDADES FÍSICAS DEL ENFERMO EN EL FINAL DE LA VIDA}

Las necesidades físicas del enfermo en el final de la vida son similares a las de cualquier paciente seriamente enfermo, en general es una progresiva falla de los diversos sistemas, a medida que se torna más débil. El tratamiento en esta fase está dirigido al control inmediato de los síntomas que deterioran la calidad de vida. Se suspenden los tratamientos que no contribuyen para nada en su alivio (ej. antibióticos y otros). En casa, el enfermo va a requerir un suplemento de drogas y acompañamiento en las 24 horas del día, a través de la observación regular y el control eficaz de los síntomas, principalmente: dolor y agitación. Algunos cuidados básicos son esenciales para mantener su bienestar, como la higiene corporal, cuidados continuos con la boca, para evitar la sequedad. El cambio de decúbito se dirige al confort del enfermo. La incontinencia y retención urinaria y fecal son problemas que necesitan de vigilancia. ${ }^{10,11}$

El enfermo al presentar dificultad en deglutir la medicación puede requerir otras vías de administración, como la subcutánea, por la necesidad de dar continuidad a terapéuticas como opioides, ansiolíticos y antieméticos. En esta fase no se recomienda la reanimación cardio-pulmonar. Cuando prestamos un buen cuidado, la mayoría de las muertes pueden ser efectivamente en paz y relativamente libres de desconfort, aunque algunos síntomas sean complicados de controlar. ${ }^{10,19}$

\section{NECESIDADES PSICOSOCIALES DEL ENFERMO EN EL FINAL DE LA VIDA}

El proceso de morir promueve alteraciones y vulnerabilidades tanto físicas, como psicológicas y espirituales/existenciales. Las alteraciones son graduales con redefinición interior y de las expectativas personales. ${ }^{4}$ La principal necesidad es la de morir con dignidad.

Las oscilaciones de los sentimientos son comunes, con respuestas emocionales marcadas, como: ruptura (incredulidad, negación, choque, desespero), disforia (ansiedad, insomnio, dificultad de concentración, cólera, culpa, cese de la actividad, tristeza, depresión) y por fin, adaptación (enfrentar las implicaciones, establecimiento de nuevas metas, esperanza restaurada con nuevos objetivos, retoma de las actividades). ${ }^{3}$ El enfermo aprenderá a desprenderse de algunas de sus capacidades y atributos, elaborando sus propias luchas, para conservar el sentido de la vida. El enfermo en esta fase debe percibir que no está solo, que continúa siendo importante y que sus cuidados se extienden hasta los últimos momentos de vida. ${ }^{5,11}$

Se recomienda la expresión de las emociones, dudas y miedos para que puedan ser resueltos. La opinión del enfermo debe ser tomada en cuenta, si es posible permanecer en el domicilio en el caso que lo desee. La familia perspicazmente, conocedora de la situación del enfermo debe evaluar y cuestionarse el proceso de morir y explorarlo de modo apropiado y sensíble. El profesional que propicie cuidados al enfermo y a la familia debe fomentar la expresión de sentimentos rompiendo la "conspiración del silencio", situación en que no se habla, omitiéndose la muerte que se aproxima pero, que todos saben que va a ocurrir. ${ }^{10,11}$

Los últimos dias de vida deben ser una etapa cuyo objetivo es obtener paz, alivio, calidad de vida y calidad en el proceso de morir. Decidir una situación de ausencia de malestar físico y 
alivio del malestar psicológico, que repercuta positivamente en la condición espiritual del enfermo y familia y/o persona significativa. ${ }^{11}$

\section{EL CUIDADOR EN CUIDADOS PALIATIVOS}

El cuidado en el final de la vida generalmente incluye el cuidado paliativo en casa, excepto cuando el enfermo muere solo o no tiene família y/o persona significativa que le proporcione el soporte y asistencia que necesita. ${ }^{16,20}$

La carga y recompensa del cuidado a enfermos en el último año de vida se divide entre cuidadores informales y formales. Los cuidadores informales son miembros de la familia, amigos o vecinos, que prestan cuidados en casa de forma no remunerada. Entre estos, más del $75 \%$ son mujeres y cerca del 33\% tienen más de 65 años. Estos cuidadores asumen una considerable carga psicológica, física y económica en el cuidado en casa. Los cuidadores formales son profesionales de salud que trabajan en instituciones 0 en la comunidad, compensados por el cuidado prestado. ${ }^{13,21}$

Muchos enfermos expresan el deseo de morir en casa, donde los miembros de la família tienen una considerable importancia en la prestación de cuidados. Estos últimos sufren las consecuencias en su propia salud, por el hecho de ser el cuidado en el final de la vida un encargo, cuya duración puede ser comparada con el cuidado a enfermos con enfermedades semejantes al Alzheimer, que duran una década o más, de fuerte impacto en la salud y bienestar de los cuidadores, llevándolos a situaciones de estrés. ${ }^{22}$ Un estudio revela que cerca del $77 \%$ de los cuidadores relatan un aumento del estrés y que el $28 \%$ requierem medicación para enfrentar la carga asociada a la prestación de cuidados. ${ }^{21}$

El enfermo en el final de la vida generalmente pasa gran parte del último año de vida en casa, aunque el $90 \%$ pase algún tiempo en el Hospital, donde ocurre cerca del $55 \%$ de todas las muertes. La razón de que gran parte de estas ocurran en un hospital se debe a la excesiva tension de los familiares, la falta de apoyo profesional, falta de referencias principalmente durante la noche y la ausencia de equipamiento. ${ }^{13}$

Lo que nos lleva a cuestionarnos: ¿La familia está preparada para lidiar con el enfermo en el final de la vida? ¿Lidiar con el dolor, el sufrimiento y proporcionar un buen control de los síntomas? ¿Lidiar con la muerte del ser querido?

Al intervenir tenemos que tener en cuenta que la enfermedad y la hospitalización promueven una ruptura en la estructura familiar, que lleva al desequilibrio, generando conflictos, distanciamiento y alteración en la vida familiar. ${ }^{23}$ Será necesario ofrecerles tiempo para ayudarlos a reflexionar, a preveer necesidades y estimular la búsqueda de recursos y soluciones. El equipo de salud puede funcionar como elemento intermediario, percibiendo sensibilidades, deseos, últimas voluntades y fomentando momentos de comunicación intensa y exposición y compartimiento de lo mejor que se tiene para dar a los más íntimos en la eminente partida. ${ }^{24}$ La adaptación progresiva a las pérdidas sucesivas llevan al cuidador a prepararse con mayor o menor dificultad para esta última pérdida, la muerte de su prójimo. $^{2}$

En la prestación del cuidado en casa el cuidador necesita de mucho apoyo y de comprensión en cuanto: a sus problemas y dificultades en el trabajo; cuidados de salud; educación e información adecuada para una buena prestación de cuidados al enfermo y sobre el diagnóstico, acerca de las causas, importancia y gestión de los síntomas; probable 
pronóstico y cómo el enfermo fallecerá; súbitos cambios en la condición del enfermo, principalmente en las señales de la aproximación de la muerte. ${ }^{13,21,25}$

Las necesidades de la familia varían de forma contínua, del inicio hasta mucho tiempo después de la muerte del familiar, siendo necesario ayudarlos a mantener el equilibrio entre ser útil al enfermo y respetar sus propias necesidades. ${ }^{1,19}$

En cuanto a la información, ésta debve ser adecuada sobre el diagnóstico, pronóstico y posibles tratamientos, lo que permite a los enfermos y cuidadores tomar decisiones acerca del tratamiento, cuidados personales y cuestiones sociales. ${ }^{13,21,26}$ Esta debe ser aportada gradualmente y siempre adaptada a la voluntad, personalidad, comprensión y necesidades de saber manifestadas por el enfermo. Lo que requiere tiempo y un proceso de adaptación del enfermo y familia. ${ }^{14}$

La educación acerca del cuidado es esencial porque el cuidador necesita de entrenamiento en cuanto a los procedimentos diarios con el enfermo, beneficiándose de las instrucciones prácticas de cómo levantarlo y administrarle la medicación. Debe presentar contenidos técnicos mínimos sobre los cuidados a prestar en el domicílio. Los cuidadores refieren sentimientos de inutilidad y desamparo cuando no son enseñados adecuadamente. ${ }^{11,13,16}$ Los cuidados generalmente prestados por los cuidadores informales van desde sencillos cuidados de higiene, a cuidados especializados, como el empleo de traqueotomias y administración terapéutica endovenosa y subcutanea. ${ }^{21}$

El soporte psicológico es esencial, porque, en el último año de vida del enfermo es estimada una alta prevalencia de ansiedad (46\%) depresión (39\%) en los cuidadores informales. Los cuidadores deben ser estimulados a exponer sus sentimentos y recelos. Alteraciones más severas requieren la intervención de un especialista. ${ }^{13}$

El mostrar disponibilidad para oir, estar, dejar hablar y exteriorizar sus ansias, forma parte de la comunicación. La comunicación es una constante durante todo el precurso. Es necesaria una comunicación discreta que relate sus necesidades, tales como la obtención de información, sintetización de la información, toma de decisiones e intentar transmitir algún sentimiento de control (autonomía). La comunicación que será necesaria para la familia u otro soporte depende de su papel dentro del sistema familiar, de su edad, de su habilidad en tomar decisionesy de las reglas dentro del seno familiar. Los familiares vivencian altos niveles de frustración y necesitan de información constante a fin de organizarse y dar prioridad a actividades de su papel de cuidador. ${ }^{27}$

Es primordial mantener una comunicación abierta con los familiares acerca de las alteraciones que puedan ocurrir durante el proceso y responder a las cuestiones de forma sincera. La familia debe entender el proceso de morir, explicándoles las señales y síntomas tales como la aproximación de la etapa final, para que estén preparados e informados del porqué de los cambios y cuándo surgirán. En la ausencia de la familia estas alteraciones deben ser informadas por teléfono y se debe respetar su ausencia. ${ }^{28}$

\section{LA CARGA DEL CUIDADOR}

El soporte al cuidador informal es relevante, ya que tienen el papel preponderante en el cuidado al enfermo en el final de la vida. El apropiado soporte los ayuda a dar prioridad y dirigir los problemas del enfermo, aunque todavía las "cargas" de ese cuidado sean ambíguos. La simple intervención de reconocimiento y apoyo reduce considerablemente la 
muerte hospitalaria y el estrés del enfermo y familia. En el caso de que la familia reconozca su valor en la prestación de cuidados, el sentido de "carga", probablemente disminuirá. ${ }^{29,30}$

El estudio de RABOW, HAUSER e ADAMS ${ }^{31}$ identifica como cargas del cuidador:

$4 \quad$ Tiempo y logística - los cuidadores pueden prestar cuidados durante algunas horas a la semana, el $20 \%$ de los cuidadores prestan cuidados a jornada completa o constante. La duración de la enfermedad, en su trayectoria hasta la muerte y declive es difícil de predecir, particularmente en enfermos no cancerosos. En esta trayectoria los familiares generalmente coordinan la administración de la numerosa terapéutica, tratamientos, servicios sociales o clínicosasí como sus propias necesidades;

$\Leftrightarrow \quad$ Tareas físicas - la tarea de cuidador muchas veces es desempeñada a personas ancianas, a enfermos y a incapacitados. Los cuidadores generalmente no tienen formación sobre la realización de cambios posturales a pacientes seriamente enfermos, además de tener que realizar arduas tareas rutinariamente, lo que lleva a un significativo riesgo de problemas físicos;

$\Leftrightarrow \quad$ Costes financieros - el enfermo a veces se vuelve una carga financiera, por ser necesario desembolsar dinero para su cuidado, así como por la pérdida del rendimiento y beneficios. La condición financiera de la familia puede alterarse profundamente, tal como un estudio revela, en que el $20 \%$ de los familiares tuvieron que dejar de trabajar y el $31 \%$ perdieron las economias de la familia como resultado de la prestación de cuidados. Este aspecto es visto de diferentes maneras, por la óptica del enfermo; éste muchas veces se considera una carga para la familia, a pesar de que ésta lo provea de cuidados con buena voluntad. La privación económica está asociada a la prioriad por las medidas de confort más que a prestar cuidados que prolonguen la vida;

$4 \quad$ Carga emocional y riesgos en la salud mental - el cuidador soporta una incalculable carga emocional, aunque muchos gocen de un profundo sentido de privilegio y profunda satisfacción por el papel que desempeñan. Otros presentan sentimientos tales como tristeza, culpa, rabia, resentimento. Reacciones comunes y comprensibles, además de sentimientos de incapacidad;

$\Leftrightarrow \quad$ Riesgos de salud - la familia tiene tendencia a colocar las necesidades del enfermo por delante de las suyas, minimizando la severidad de sus problemas y olvidándose 0 tardando en resolverlos. Los cuidadores presentan un particular riesgo en lo que concierne a la manutención de los niveles de salud y pérdida significativa en el tiempo social y de placer. Está relacionado el aumento de la mortalidad del cuidador, por el hecho de que los cuidadores están sujetos a gran tensión mental y emocional, con un riesgo de mortalidad del $63 \%$.

\section{CONSIDERACIONES FINALES}

Las medidas implementadas en el final de la vida tienden al confort del enfermo. Cuando este proceso ocurre de manera digna y controlada y sin sufrimiento, habrá una mayor aceptación de la familia; en caso de que el proceso sea muy negativo puede repercutir en la familia y/o persona significativa, bajo la forma de un trauma presente a lo largo de toda la vida. 
El sufrimiento de quien muere puede ser aminorado por los que cuidan, simplemente con el mirar, con la forma de estar, por el gesto lleno de respeto y ternura. El proceso de muerte no se puede estancar en el tiempo... es un pasaje obligatorio, por eso cuidar de alguien que parte es ayudarlo en esa travesía, acompañándolo hasta el umbral de la puerta, sabiendo decirle adiós.

Los cuidados paliativos nos enseñan que puede haber un límite para la cura, pero no hay límite para los cuidados. Como KÜBLER-ROSS refiere “... podemos ayudarlos a morir, intentando ayudarlos a vivir..." (pág. 33).

\section{REFERENCIAS BIBLIOGRÁFICAS}

1.KÜBLER-ROSS, E. Sobre a Morte e o Morrer. 7ª Edição, São Paulo: Martins Fontes, 1996. 2.Sociedade Francesa de Acompanhamento e de Cuidados Paliativos. Cuidar e Organizarse. In: Desafios da Enfermagem em Cuidados Paliativos - Cuidar: ética e práticas. Loures: Lusociência 1999; 77-136.

3.TWYCROSS, R. Cuidados Paliativos. 2ª Edição, Lisboa: Climepsi 2003. 13-179.

4.CHOCHINOV, H.M. Dying, Dignity, and New Horizons in Palliative End-of-Life Care. A Cancer Journoul for Clinicians 2006; 56:84-103.

5.LEVIN, R. Cuidados Paliativos: Principios Generales. In: Cuidados Paliativos: Guías para el Manejo Clínico. Organización Panamericana de la Salud; 3-8. http://www.paho.org/sapanish/AD/DPC/NC/palliative-care.pdef - visitado em 18 de Dezembro de 2005.

6.HIGGINSON, I.J. Evidence Based Palliative Care. BMJ 1999; 319:462-463.

7.MONGE,M.A.; CASTRO, P.; La Muerte, Final de la Vida Humana. In: Medicina Pastoral. Pamplona, Edições Universidade de Navarra; 2002: 189-222.

8.GUTEN,C.F.V. Interventions to Manage Symptoms at the End of Life. Journal of Palliative Medicine 2005; 8(1): 88-94.

9.WATSON, M. LUCAS, C.; et al. The Terminal Phase. In: Oxford Handbook of Palliative Care. New York: Oxford University 2005; 735-745.

10.ELLERSHAW, J; WARD, C. Care of the Dying Patient: The Last Hours or Days of Life. BMJ 2003; 326:30-34.

11.ROSÁRIO, M.A.B.; PASCUAL,L.; FRAILE,A.A. La Atención a los Últimos Días. Aten Primaria 2002; 30(5): 318-322.

12.GONÇALVES, J.F; ALVARENGA, M; SILVA, A. The Last Forty-Eight Hours of Life in a Portuguese Palliative Care Unit: Does it differ from Elsewhere? Journal of Palliative Medicine 2003; 6 (6): 895-900.

13.RAMIREZ, A.; ADDINGTON-HALL, J.; RICHARDS M. ABC of Palliative Care - The Carers. BMJ 1998; 316 (17): 208-211.

14.PIRES, A.M.L. Cuidar em Fim-de-Vida: Aspectos Éticos. In: Cadernos de Bioética. Coimbra; Edição do Centro de Estudos Bioéticos, 2001; 25:85-94.

15.MANSO,F.R.; VELHO,L.V. Fim de Vida e Suas Implicações. Nursing 2004; 186:24-28.

16.HUGHES,R.G.; BAKOS,A.D et al. Palliative Wound Care at the End of Life. Home Health Care Management \& Practice 2005; 17 (3): 196-202.

17.PÉREZ, C.G. La Enferméria en Cuidados Paliativos. In: Enfermería en Cuidados Paliativos, Editorial Médica Panamericana, Madrid 1998: 31-34.

18.ECHEVERRI,T.A. El Cuidado Paliativo en Casa al Paciente Terminal. MEDUNAB 2001; 4 (10): 1-11.

19.HALLENBECK, J. Palliative Care in the Final Days of Life. JAMA 2005; 293 (18): 22652271.

20.STAJDUHAR, K.I. Examining the Perspectives of Family Members Involved in the Delivery 
of Paliative Care at Home. Journal of Palliative Care 2003; 19 (1): 27-35.

21.MILONE-NUZZO, P.; McCORKLE,R. Home Care. In: Ferrel, B.R.; Coylen, N. Textbook of Palliative Nursing, 2a Edição, New York, Oxford University Press, Inc 2004: 771-785.

22.ZARIT, S.H. Family Care and Burden at the End of Life. JAMC 2004; 170 (12): 1811-1812. 23.PETTENGILL, M.A.M.; ANGELO,M. Vulnerabilidade da Família: Desenvolvimento de um Conceito. Revista Latino-am Enfermagem 2005;13 (6):982-988.

24.SOARES, M.L.B. O Doente Terminal, a Morte Anunciada. In: Cadernos de Bioética. Coimbra; Edição do Centro de Estudos de Bioética; 2002; 28: 89-94.

25.HARDING, R. Clinical Notes for Informal Carers in Palliative Care: Recommendations From a Random Patient File Audit. Palliative Medicine 2005; 19: 639-642.

26.LEYDON,G.M.; BOULTON,M. et al. Cancer Patients' Information Needs and Information Seeking Behaviour: in depth interview study. BMJ 2000; 320 (1): 909-913.

27. DAHLIN, C.M.; GIANSIRACUSA, D.F. Communication in Palliative Care. In: Ferrel, B.R.; Coylen, N. Textbook of Palliative Nursing, 2ª Edição, New York, Oxford University Press, Inc 2004: 67-93.

28. WILSON, S. Long-Term Care. In: Ferrel, B.R.; Coylen, N. Textbook of Palliative Nursing, 2a Edição, New York, Oxford University Press, Inc 2004: 759-769.

29.GIVEN, B.A.; GIVEN, C.W.; KOZACHIK. Family Support in Advanced Cancer. A Cancer Journal for Clinicians, 2001; 51:213-231.

30.BRUERA, E. Improving the Care of Terminally III Cancer Patients and Their Families. Journal of Clinical Oncology, 2005; 23 (30): 7378-7379.

31. RABOW, M.W.; HAUSER, J.M.; ADAMS J. Supporting Family Caregivers at the End of Life. JAMA, 2004; 291 (4): 483-492. 